\title{
Testing a direction-dependent primordial power spectrum with observations of the Cosmic Microwave Background
}

\author{
Yin-Zhe Ma, ${ }^{1, *}$ George Efstathiou, ${ }^{1, \dagger}$ and Anthony Challinor ${ }^{1,2, \ddagger}$ \\ ${ }^{1}$ Kavli Institute for Cosmology at Cambridge and Institute of Astronomy, \\ Madingley Road, Cambridge CB3 OHA, United Kingdom \\ ${ }^{2}$ DAMTP, Centre for Mathematical Sciences, \\ Wilberforce Road, Cambridge CB3 OWA, United Kingdom
}

\begin{abstract}
Statistical isotropy is often assumed in cosmology and should be tested rigorously against observational data. We construct simple quadratic estimators to reconstruct asymmetry in the primordial power spectrum from CMB temperature and polarization data and verify their accuracy using simulations with quadrupole power asymmetry. We show that the Planck mission, with its millions of signal-dominated modes of the temperature anisotropy, should be able to constrain the amplitude of any spherical multipole of a scale-invariant quadrupole asymmetry at the 0.01 level $(2 \sigma)$. Almost independent constraints can be obtained from polarization at the 0.03 level after four full-sky surveys, providing an important consistency test. If the amplitude of the asymmetry is large enough, constraining its scale-dependence should become possible. In scale-free quadrupole models with $1 \%$ asymmetry, consistent with the current limits from WMAP temperature data (after correction for beam asymmetries), Planck should constrain the spectral index $q$ of power-law departures from asymmetry to $\Delta q=0.3$. Finally, we show how to constrain models with axisymmetry in the same framework. For scale-free quadrupole models, Planck should constrain the direction of the asymmetry to a $1 \sigma$ accuracy of about 2 degrees using one year of temperature data.
\end{abstract}

\footnotetext{
*Electronic address: yzm20@cam.ac.uk

$\dagger$ Electronic address: gpe@ast.cam.ac.uk

${ }^{\ddagger}$ Electronic address: a.d.challinor@ast.cam.ac.uk
} 


\section{INTRODUCTION}

In recent years, observations of the cosmic microwave background (CMB) radiation fluctuations by the Wilkinson Microwave Anisotropy Probe (WMAP) and a large number of ground-based and suborbital experiments have led to a precise measurement of the temperature anisotropy power spectrum up to multipoles of a few thousand ([1-6]) . Apart from some claimed "anomalies" (see below) the observations are consistent with a darkenergy-dominated cosmology with statistically isotropic, Gaussian, adiabatic perturbations, as expected from simple models of inflation. (We will refer to this as the "concordance" $\Lambda \mathrm{CDM}$ model.) If statistical isotropy applies, then the harmonic coefficients of the temperature field,

$$
a_{l m}^{T}=\int d \Omega Y_{l m}^{*}(\Omega) \Delta T(\Omega)
$$

must satisfy

$$
C_{l m, l^{\prime} m^{\prime}}^{T T}=\left\langle a_{l m}^{T} a_{l^{\prime} m^{\prime}}^{T *}\right\rangle=C_{l}^{T T} \delta_{l l^{\prime}} \delta_{m m^{\prime}}
$$

If the fluctuations are Gaussian and statistically isotropic, their statistical properties are completely described by the power spectrum $C_{l}^{T T}$.

There have been some hints of "anomalies" in the WMAP data, perhaps suggesting a violation of statistical isotropy. These include alignments of low- $l$ multipoles [7-10], evidence for power asymmetry $[11,12]$ and for a deep cold spot in the southern Galactic hemisphere $[13,14]$. For the most part, these anomalies have been found by examining the data without reference to specific theoretical models. There is, therefore, an a posteriori aspect in computing their statistical significance which is difficult to assess [15]. Some authors have, however, claimed highly significant discrepancies between the CMB data and the concordance $\Lambda$ CDM model [16].

Interest in the CMB anomalies has motivated theorists to build inflationary models that violate rotational invariance, either via the addition of a vector field [17-19] or via an isocurvature perturbation $[20,21]$. In addition, a number of phenomenological models that violate statistical isotropy have been proposed, which can be tested against observations (e.g. $[17,22,23])$.

Reference [17] considers the phenomenological model in which the primordial power spec- 
trum depends on a preferred direction,

$$
\mathcal{P}(\mathbf{k})=\mathcal{P}(k)\left[1+g(k)(\mathbf{k} \cdot \mathbf{n})^{2}\right],
$$

where $g(k)$ is some arbitrary function of wavenumber. There has been considerable interest in this model recently. The authors of [24] applied Gibbs sampling to the WMAP five-year maps to test models with $g(k)=g_{*}=$ constant, finding strong evidence for a preferred direction with $g_{*}=0.15 \pm 0.039$ using multipoles $l \leq 400$. Hanson and Lewis [25] corrected some algebraic errors in the analysis of [24] and applied a simpler quadratic estimator to the WMAP 5-year data. These authors also found evidence for a highly significant $(9 \sigma)$ departure from statistical isotropy, but with a preferred direction suspiciously close to the ecliptic poles, suggestive of some type of systematic effect in the WMAP data. This analysis was confirmed by [26]. A subsequent analysis [27] showed that asymmetries in the WMAP beams fully account for the observed violation of statistical isotropy.

Despite this negative conclusion, it is still important to assess the prospects of constraining violations of statistical isotropy in the CMB with more precise experiments. In this paper, we focus on constraints from the Planck satellite, which was launched successfully in May 2009 and has recently completed its third full scan of the sky. The Planck satellite has much higher signal-to-noise ratio in both temperature and polarization than WMAP [28]. It also has higher angular resolution -5 arcmin full-width at half-maximum (FWHM) at frequencies $\geq 217 \mathrm{GHz}$ - so asymmetries on scales of the beam width should have little effect at multipoles $l \lesssim 1000$. It is also expected that the Planck beams will be calibrated to high precision from scans of bright planets [29]. Following the successful launch of Planck, the European Space Agency (ESA) has approved a mission extension until the on-board cryogens are depleted. Planck is therefore expected to produce almost five sky surveys, compared to the two sky surveys approved for the nominal mission. This combination of high sensitivity, high resolution and extended lifetime allows greater scope for testing for systematic effects than was possible with WMAP. For example, it becomes possible to use polarization maps independently of temperature maps to test for violations of statistical isotropy.

In this paper, we extend the analysis of [30] to assess how accurately an extended Planck mission can be used to test models with an anisotropic primordial power spectrum. This paper is organized as follows: In Section II, we summarize some basic properties of the 
anisotropic model. Section III then applies the quadratic estimator of [25], extended to include polarization, to compute forecasts for Planck. Our conclusions are presented in Section IV.

\section{THE ANISOTROPIC MODEL}

\section{A. Covariance matrix}

We write the anisotropic primordial power spectrum as

$$
\mathcal{P}(\mathbf{k})=\mathcal{P}(k)\left(1+\sum_{L M} g_{L M}(k) Y_{L M}(\hat{\mathbf{k}})\right) .
$$

We assume that parity-invariance continues to hold in the mean so that $L$ is restricted to even values such that $\mathcal{P}(-\mathbf{k})=\mathcal{P}(\mathbf{k})$. In this paper, we consider a quadrupole modulation, i.e. $L=2,|M| \leq 2$, with a power-law scale dependence on the wave number $g_{L M}(k)=$ $g_{L M}\left(k_{0} / k\right)^{q}$ where the pivot scale $k_{0}=0.002 \mathrm{Mpc}^{-1}$. Scale-invariant modulation corresponds to $q=0$.

The harmonic coefficients of the CMB anisotropy can be expressed as

$$
a_{l m}^{X}=4 \pi i^{l} \int \frac{d^{3} \mathbf{k}}{(2 \pi)^{3}} \Delta_{l}^{X}(k) \mathcal{R}(\mathbf{k}) Y_{l m}^{*}(\hat{\mathbf{k}})
$$

where $\Delta_{l}^{X}(k)$ are the adiabatic transfer functions, either for temperature $(X=T)$ or $E$-mode polarization $(X=E)$. The primordial curvature perturbation is $\mathcal{R}(\mathbf{k})$ with statisticallyhomogeneous but anisotropic correlations

$$
\left\langle\mathcal{R}(\mathbf{k}) \mathcal{R}^{*}\left(\mathbf{k}^{\prime}\right)\right\rangle=(2 \pi)^{3} \delta^{3}\left(\mathbf{k}-\mathbf{k}^{\prime}\right) \frac{2 \pi^{2}}{k^{3}} \mathcal{P}(\mathbf{k}),
$$

with $\mathcal{P}(\mathbf{k})$ given by Eq. (4). Thus, the covariance matrix of the harmonic coefficients is

$$
\begin{aligned}
C_{l_{1} m_{1}, l_{2} m_{2}}^{X X^{\prime}} & =\left\langle a_{l_{1} m_{1}}^{X} a_{l_{2} m_{2}}^{X^{\prime} m_{2}}\right\rangle \\
& =C_{l_{1}}^{X X^{\prime}} \delta_{l_{1} l_{2}} \delta_{m_{1} m_{2}}+\delta C_{l_{1} m_{1}, l_{2} m_{2}}^{X X^{\prime}}
\end{aligned}
$$

where

$$
C_{l_{1}}^{X X^{\prime}}=4 \pi \int d \ln k \mathcal{P}(k) \Delta_{l_{1}}^{X}(k) \Delta_{l_{1}}^{X^{\prime}}(k)
$$


is the usual isotropic power spectrum. The additional term in Eq. (7) due to the power asymmetry is

$$
\begin{aligned}
\delta C_{l_{1} m_{1}, l_{2} m_{2}}^{X X^{\prime}}= & i^{l_{1}-l_{2}} \tilde{C}_{l_{1} l_{2}}^{X X^{\prime}}(q) \sum_{L M} g_{L M} \int d \Omega_{k} Y_{L M}(\hat{\mathbf{k}}) Y_{l_{1} m_{1}}^{*}(\hat{\mathbf{k}}) Y_{l_{2} m_{2}}(\hat{\mathbf{k}}) \\
= & i^{l_{1}-l_{2}} \tilde{C}_{l_{1} l_{2}}^{X X^{\prime}}(q) \sum_{L M} g_{L M} \\
& \times(-1)^{m_{1}}\left[\frac{(2 L+1)\left(2 l_{1}+1\right)\left(2 l_{2}+1\right)}{4 \pi}\right]^{\frac{1}{2}}\left(\begin{array}{ccc}
L & l_{1} & l_{2} \\
0 & 0 & 0
\end{array}\right)\left(\begin{array}{ccc}
L & l_{1} & l_{2} \\
M & -m_{1} & m_{2}
\end{array}\right)
\end{aligned}
$$

where

$$
\tilde{C}_{l_{1} l_{2}}^{X X^{\prime}}(q)=4 \pi \int d \ln k \mathcal{P}_{\mathcal{R}}(k) \Delta_{l_{1}}^{X}(k) \Delta_{l_{2}}^{X^{\prime}}(k)\left(\frac{k_{0}}{k}\right)^{q} .
$$

Since $L$ is even, the anisotropic covariance is nonzero only for $l_{1}-l_{2}$ even as required by parity invariance. In the following it will also be convenient to introduce $2 \times 2$ matrices $\mathbf{C}_{l_{1} m_{1}, l_{2} m_{2}}$ and $\tilde{\mathbf{C}}_{l_{1} l_{2}}$ with elements $C_{l_{1} m_{1}, l_{2} m_{2}}^{X_{1} X_{2}}$ and $\tilde{C}_{l_{1} l_{2}}^{X_{1} X_{2}}(q)$ respectively.

\section{B. Quadratic estimators and the Fisher matrix}

Here we assume that the scale-dependence of the power asymmetry (i.e. $q$ ) is known. We can then use the quadratic estimator of Ref. [25], extended to polarization, to form estimates $\hat{g}_{L M}$ of the anisotropy parameters. For an isotropic survey and in the limit of small primordial anisotropy, these take the form

$$
\hat{g}_{L M}=\frac{1}{2} \sum_{L^{\prime} M^{\prime}} F_{L M, L^{\prime} M^{\prime}}^{-1} \sum_{X_{1} l_{1} m_{1}} \sum_{X_{2} l_{2} m_{2}} \bar{a}_{l_{1} m_{1}}^{X_{1} *} \frac{\partial C_{l_{1} m_{1}, l_{2} m_{2}}^{X_{1} X_{2}}}{\partial g_{L^{\prime} M^{\prime}}^{*}} \bar{a}_{l_{2} m_{2}}^{X_{2}} .
$$

Here, $\bar{a}_{l m}^{X} \equiv \sum_{X^{\prime}}\left[\left(\mathbf{C}_{l}^{\text {tot }}\right)^{-1}\right]^{X X^{\prime}} a_{l m}^{X^{\prime}}$ are the temperature and polarization multipoles after weighting with the inverse of their isotropic total (signal-plus-noise) covariance matrix. The Fisher matrix, evaluated at $g_{L M}=0$, is given by

$$
F_{L M, L^{\prime} M^{\prime}}=\frac{1}{2} \sum_{l_{1} m_{1}} \sum_{l_{2} m_{2}} \operatorname{Tr}\left[\left(\mathbf{C}_{l_{1}}^{\mathrm{tot}}\right)^{-1} \frac{\partial \mathbf{C}_{l_{1} m_{1}, l_{2} m_{2}}}{\partial g_{L M}^{*}}\left(\mathbf{C}_{l_{2}}^{\mathrm{tot}}\right)^{-1} \frac{\partial \mathbf{C}_{l_{2} m_{2}, l_{1} m_{1}}}{\partial g_{L^{\prime} M^{\prime}}}\right] .
$$

In the limit of vanishing primordial anisotropy, the inverse of this Fisher matrix equals the covariance of the errors on $\hat{g}_{L M}$, i.e. $F_{L M, L^{\prime} M^{\prime}}^{-1}=\left\langle\hat{g}_{L M} \hat{g}_{L^{\prime} M^{\prime}}^{*}\right\rangle$.

The assumed isotropy of the survey ensures that the Fisher matrix at $g_{L M}=0$ is diagonal. 
Using Eq. (9), the diagonal elements evaluate to

$$
F_{L M, L M}=\sum_{l_{1} l_{2}}\left[\operatorname{Tr}\left(\tilde{\mathbf{C}}_{l_{1} l_{2}}\left(\mathbf{C}_{l_{2}}^{\text {tot }}\right)^{-1} \tilde{\mathbf{C}}_{l_{2} l_{1}}\left(\mathbf{C}_{l_{1}}^{\text {tot }}\right)^{-1}\right) \frac{\left(2 l_{1}+1\right)\left(2 l_{2}+1\right)}{8 \pi}\left(\begin{array}{ccc}
l_{1} & l_{2} & L \\
0 & 0 & 0
\end{array}\right)^{2}\right]
$$

Since $g_{L M}$ is complex for $M \neq 0$, we present our simulation results in the next section in terms of $\hat{G}_{L M}=\sqrt{2} \operatorname{Re}\left(\hat{g}_{L M}\right)$ and $\hat{G}_{L-M}=\sqrt{2} \operatorname{Im}\left(\hat{g}_{L M}\right)$ for $M>0$, and $\hat{G}_{L 0}=\hat{g}_{L 0}$. The $\hat{G}_{L M}$ are uncorrelated and have the same variance as the $\hat{g}_{L M}$.

\section{FORECASTS FOR PLANCK}

\section{A. Constraints on the anisotropy amplitude}

In this section, we consider forecasts for the Planck mission. We use the parameters for the $143 \mathrm{GHz}$ channel (the most sensitive of the Planck frequency channels) as given in [28]. We therefore assume a Gaussian beam with FWHM of 7.1 arcmin and assume uncorrelated isotropic noise in the temperature and polarization maps with root-mean-square noise levels of $\sigma_{T}$ and $\sigma_{P}$ respectively. For the nominal two-sky-survey mission (one year of observation) we adopt $\sigma_{T}=12.2 \mu \mathrm{K}$ and $\sigma_{P}=23.3 \mu \mathrm{K}$ in 3.4 arcmin pixels (Healpix [31] resolution $\left.N_{\text {side }}=1024\right)$, corresponding to $42 \mu \mathrm{K}$-arcmin noise in temperature and $80 \mu \mathrm{K}$-arcmin in Stokes $Q$ and $U$ polarization. We also consider an extended mission of four complete sky surveys (two years of observation) with $\sigma_{T}$ and $\sigma_{P}$ reduced by a factor of $\sqrt{2}$.

To give a feel for the nature of the anisotropy signal, Figs. 1 and 2 show simulated sky maps for a noise-free realisation of a scale-invariant $(q=0)$ quadrupole-modulation model with $g_{20}=0.1\left(g_{2 M}=0, M \neq 0\right)$. These maps have been generated using the prescription described in [25], generalized to polarization. This uses an approximate square root of the anisotropic covariance matrix, linear in the $g_{L M}$, to simulate maps as the sum of a statistically isotropic part and an anisotropic part. The isotropic component of a noisefree temperature map is shown on the left in Fig. 1 and the anisotropic component, clearly showing a preferred direction along the polar axis, is shown on the right.

The anisotropic contribution to the Stokes $Q$ and $U$ polarization maps is shown in Fig. 2. Here, we have smoothed the polarization maps with a Gaussian of FWHM 3 deg. to en-

hance the visual impact of the statistical anisotropy in the $Q$ Stokes map. Because of the quadrupole asymmetry in the primordial power, modes of the primordial perturbation with 

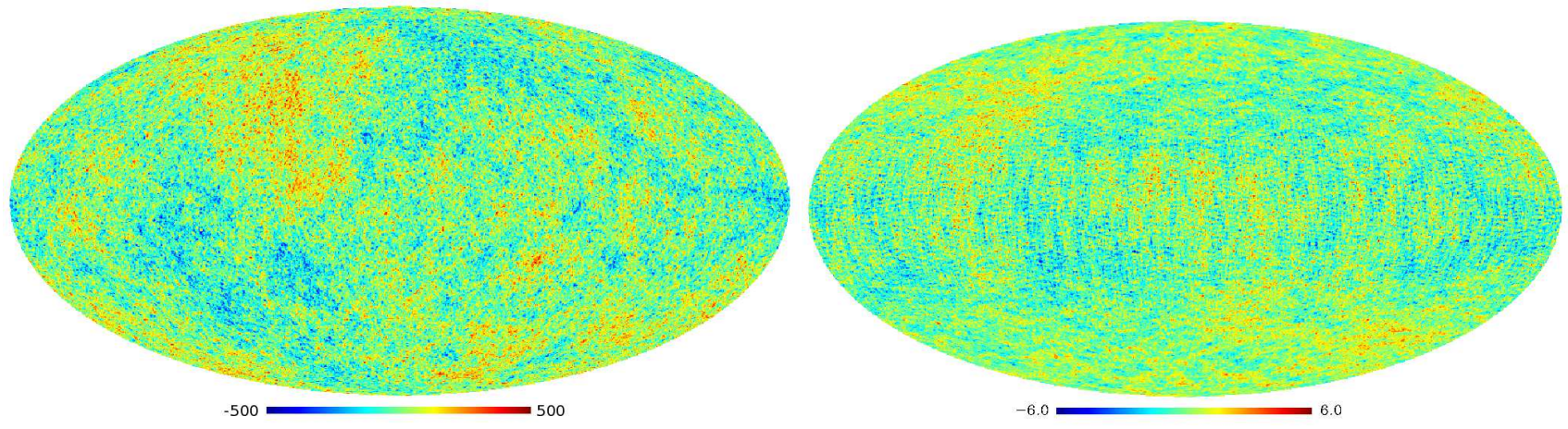

FIG. 1: Noise-free simulation of a model with scale-invariant quadrupole asymmetry in the primordial power with $g_{L M}=0.1 \delta_{L 2} \delta_{M 0}$. The isotropic component of the temperature map is shown on the left, and the anisotropic component on the right. The colour scales are in $\mu \mathrm{K}$.
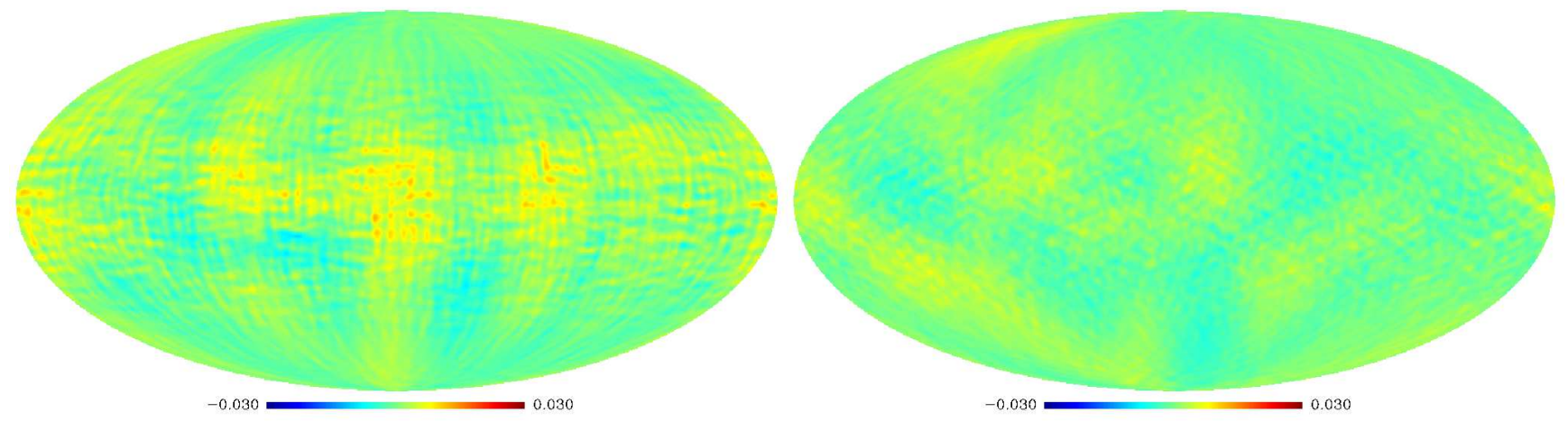

FIG. 2: Anisotropic components of the $Q$ (left) and $U$ (right) polarization maps in a noise-free simulation of the model in Fig. 1. The maps have been smoothed here with a Gaussian beam of FWHM $3^{\circ}$ to enhance the imprint of the preferred axis in the $Q$ map. The colour scales are in $\mu \mathrm{K}$.

wavevectors along the polar axis tend to have their amplitude enhanced. For such modes, the polarization generated by Thomson scattering is pure $Q$ in the polar basis and varies in amplitude with the polar angle as $\sin ^{2} \theta$ [32]. The dominant effect of the statistical anisotropy is therefore observed in the $Q$ Stokes parameter and is concentrated toward the equatorial plane.

To illustrate the machinery summarised in Section II B, we have generated five simulations of the scale-invariant quadrupole-modulation model with $g_{2 M}=0.1 \delta_{0 M}$ and added instrumental noise appropriate to one year of observation with the Planck $143 \mathrm{GHz}$ channel. 

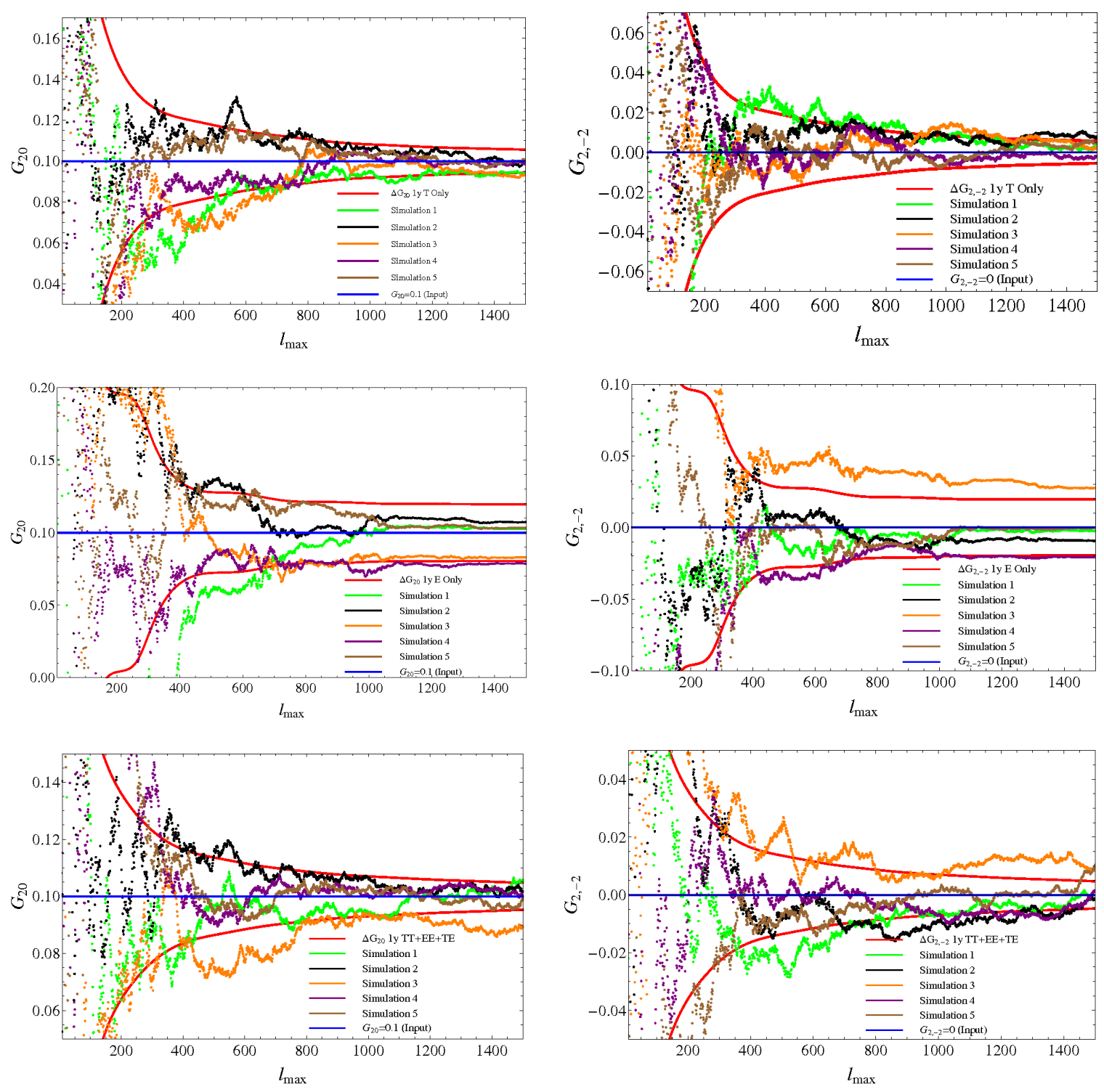

FIG. 3: Estimates of the $G_{20}$ (left) and $G_{2-2}$ (right) anisotropy parameters (shown with points) and their (one-sigma) Fisher errors ([Red] solid lines) as a function of $l_{\max }$ from five simulations of the model in Fig. 1 for one year of Planck data. The input parameters $G_{20}=0.1$ and $G_{2-2}$ are shown with horizontal [Blue] solid lines. From top to bottom we analyse temperature only, $E$-mode polarization only and temperature plus polarization.

We then estimate $g_{2 M}$ via Eq. (11) as a function of $l_{\max }$, the maximum multipole retained in the analysis. Since the only nonzero coefficient in these simulations is $g_{20}$, and the survey is assumed isotropic, the recovered estimates $\hat{g}_{2 M}$ are statistically equivalent for $M \neq 0$ and so we show results only for the two (real) components $\hat{G}_{20}$ and $\hat{G}_{2-2}$ in Fig. 3. We anal- 
yse temperature alone (top panels), E-mode polarization alone (middle), and both jointly (bottom).

With temperature alone, the errors on $G_{2 M}$ decrease approximately as $1 / l_{\max }$ over the range plotted in Fig. $3(l \leq 1500)$ reaching 0.005 by $l_{\max }$ (in agreement with the minimumvariance estimators of [30]). This behaviour follows from simple mode-counting since the temperature maps are signal-dominated over this multipole range. ${ }^{1}$ However, the polarization maps are noise-dominated over much of this multipole range and so the errors approach constant values for $l_{\max } \gtrsim 600$. Nevertheless, the Planck polarization maps alone can provide (almost) independent constraints on an anisotropic modulation to the temperature maps. For two sky surveys, the errors on the $g_{2 M}$ from polarization are four times worse than in temperature. Consistency between temperature and polarization constraints would provide an important test of systematic effects should Planck show any evidence of an anisotropic power spectrum.

\section{B. Constraints on scale-dependence}

In Fig. 4, we compare the Fisher errors on the amplitude of the modulation for a scaleinvariant model $(q=0)$ and two models with scale-dependence $(q=1$ and $q=2)$. For larger $q$, the asymmetry in the variance of the Fourier modes is confined to larger scales and so relatively more of the constraining power derives from low- $l$ multipole moments. The low- $l$ modes of polarization are enhanced by scattering at reionization [33] and are expected to be signal-dominated in the one-year Planck data. The polarization constraints on the $g_{2 M}$ therefore become more comparable to those from the temperature as $q$ increases and the improvement from observing for longer in polarization lessens.

If the amplitude of any primordial power asymmetry is high enough, it might be possible to constrain the scale-dependence of the asymmetry with Planck. To forecast constraints on the spectral index of the power asymmetry, $q$, we extend the Fisher matrix analysis of Sec. II B to include $q$ as a parameter. We must now evaluate the Fisher matrix at nonzero $g_{L M}$ but we assume that the asymmetry is still small enough that we can neglect asymmetry

\footnotetext{
${ }^{1}$ For modes that are signal-dominated, the scale dependence of the trace term in the Fisher matrix, Eq. (13), is weak. Treating the trace as constant gives Fisher information varying as $l_{\max }^{2}$ which is proportional to the number of modes retained in the analysis.
} 

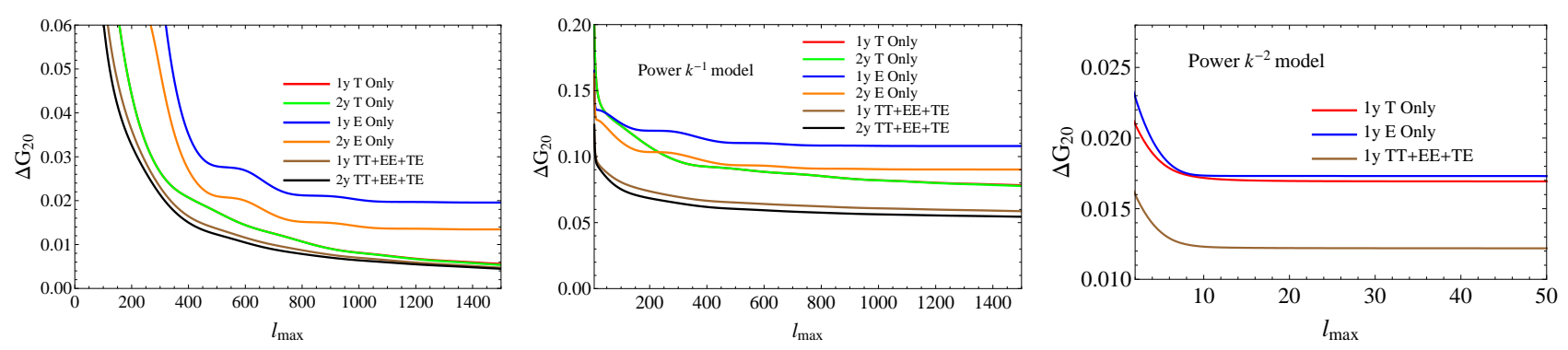

FIG. 4: Fisher errors for $G_{20}$ from temperature, $E$-mode polarization, and temperature plus polarization in models with power-asymmetry spectral indices $q=0$ (left), $q=1$ (middle) and $q=2$ (right). For $q=0$ and $q=1$ we show results for one and two years of observations; for $q=2$ we show only the one-year errors since they improve very little with further observing time. Note that the one- and two-year errors from temperature alone are indistinguishable when $q \geq 0$.

in the $\left(\mathbf{C}^{\text {tot }}\right)^{-1}$ terms. The $F_{L M, L^{\prime} M^{\prime}}$ is then unchanged from Eq. (13) but the additional elements are

$$
\begin{aligned}
F_{L M, q}= & \frac{1}{2} \sum_{l_{1} m_{1}} \sum_{l_{2} m_{2}} \operatorname{Tr}\left[\left(\mathbf{C}_{l_{1}}^{\mathrm{tot}}\right)^{-1} \frac{\partial \mathbf{C}_{l_{1} m_{1}, l_{2} m_{2}}}{\partial g_{L M}^{*}}\left(\mathbf{C}_{l_{2}}^{\mathrm{tot}}\right)^{-1} \frac{\partial \mathbf{C}_{l_{2} m_{2}, l_{1} m_{1}}}{\partial q}\right] \\
= & \sum_{l_{1} l_{2}}\left[\operatorname{Tr}\left(\tilde{\mathbf{C}}_{l_{1} l_{2}}\left(\mathbf{C}_{l_{2}}^{\mathrm{tot}}\right)^{-1} \partial_{q} \tilde{\mathbf{C}}_{l_{2} l_{1}}\left(\mathbf{C}_{l_{1}}^{\mathrm{tot}}\right)^{-1}\right) g_{L M} \frac{\left(2 l_{1}+1\right)\left(2 l_{2}+1\right)}{8 \pi}\left(\begin{array}{ccc}
l_{1} & l_{2} & L \\
0 & 0 & 0
\end{array}\right)^{2}\right](1, \\
F_{q, q}= & \frac{1}{2} \sum_{l_{1} m_{1}} \sum_{l_{2} m_{2}} \operatorname{Tr}\left[\left(\mathbf{C}_{l_{1}}^{\mathrm{tot}}\right)^{-1} \frac{\partial \mathbf{C}_{l_{1} m_{1}, l_{2} m_{2}}}{\partial q}\left(\mathbf{C}_{l_{2}}^{\mathrm{tot}}\right)^{-1} \frac{\partial \mathbf{C}_{l_{2} m_{2}, l_{1} m_{1}}}{\partial q}\right] \\
= & \sum_{l_{1} l_{2}}\left[\operatorname{Tr}\left(\partial_{q} \tilde{\mathbf{C}}_{l_{1} l_{2}}\left(\mathbf{C}_{l_{2}}^{\mathrm{tot}}\right)^{-1} \partial_{q} \tilde{\mathbf{C}}_{l_{2} l_{1}}\left(\mathbf{C}_{l_{1}}^{\mathrm{tot}}\right)^{-1}\right) \frac{\left(2 l_{1}+1\right)\left(2 l_{2}+1\right)}{8 \pi}\right. \\
& \left.\times \sum_{L M}\left|g_{L M}\right|^{2}\left(\begin{array}{ccc}
l_{1} & l_{2} & L \\
0 & 0 & 0
\end{array}\right)^{2}\right]
\end{aligned}
$$

Note that these elements vanish if $g_{L M}=0$. From the scaling of the Fisher matrix elements with $g_{L M}$, we expect the error on $q$ to scale as the inverse of the amplitude of the asymmetry.

As an example, we consider a fiducial model with scale-invariant quadrupole asymmetry with $G_{2 M}=0.03 \delta_{M 0}$. Such a model is compatible with the constraints on quadrupole asymmetry from the beam-corrected analysis of WMAP data in Ref. [27], but the amplitude should be detectable with Planck at the $6 \sigma$ level (fixing $q=0$ ). Forecasts for the (marginalised) errors on $q$ for this model are given in Table I. With temperature and polarization, Planck should constrain the spectral index to a $1 \sigma$ accuracy of $\Delta q \sim 0.3$. The 


\begin{tabular}{c|c|c|c}
\hline$\Delta q(1 \sigma)$ & $T$ only & $E$ only & $T$ and $E$ \\
\hline \hline One year & 0.399 & 1.300 & 0.322 \\
\hline Two years & 0.389 & 0.878 & 0.299 \\
\hline
\end{tabular}

TABLE I: Fisher errors on the scale-dependence of the power asymmetry assuming a scale-invariant quadrupole asymmetry with $g_{2 M}=0.03 \delta_{M 0}$.

marginalised errors on the $g_{L M}$ are similar to the case in which $q$ is fixed.

\section{Axisymmetric models}

The preceding analysis makes no assumptions about axisymmetry of the primordial power asymmetry. However, if we have good reason to expect axisymmetry, so the model is described by a preferred axis $\hat{\mathbf{m}}$ and the $M=0$ multipoles $\left\{g_{* L}\right\}$ in a frame with the polar axis along $\hat{\mathbf{m}}$, we can constrain these parameters by post-processing our estimates $\hat{g}_{L M}$. We illustrate how this works, assuming a fixed scale dependence for the primordial asymmetry.

For models with a nearly scale-invariant anisotropy spectrum, many small-scale modes contribute to the $\hat{g}_{L M}$, so we might expect the statistics of the $\hat{g}_{L M}$ to be approximately Gaussian. Expressing $\hat{\mathbf{m}}$ in terms of its azimuthal angle $\alpha$ and polar angle $\beta, \hat{\mathbf{m}}=D(\alpha, \beta, 0) \hat{\mathbf{z}}$ (i.e. a rotation of the $\hat{\mathbf{z}}$ direction through Euler angles $\alpha$ and $\beta$ ), in the Gaussian approximation we can write $\operatorname{Pr}\left(\left\{\hat{g}_{L M}\right\} \mid \hat{\mathbf{m}},\left\{g_{* L}\right\}\right) \propto \exp \left(-\chi^{2} / 2\right)$ where

$$
\chi^{2}=\sum_{L M} \sum_{L^{\prime} M^{\prime}}\left(\hat{g}_{L M}^{*}-\tilde{g}_{L M}^{*}\right) F_{L M, L^{\prime} M^{\prime}}\left(\hat{g}_{L^{\prime} M^{\prime}}-\tilde{g}_{L^{\prime} M^{\prime}}\right)
$$

Here,

$$
\tilde{g}_{L M} \equiv D_{M 0}^{L}(\alpha, \beta, 0) g_{* L}
$$

are the multipoles of the primordial asymmetry rotated from their preferred frame. $\left(D_{M M^{\prime}}^{L}(\alpha, \beta, \gamma)\right.$ are the Wigner rotation matrices.) If we now assign a uniform prior on the direction $\hat{\mathbf{m}}$ [so that $\operatorname{Pr}(\alpha, \beta) d \alpha d \beta=(4 \pi)^{-1} d \alpha d \cos \beta=(4 \pi)^{-1} d \hat{\mathbf{m}}$ ] and a flat prior on the $g_{* L}$, Bayes' theorem gives for the posterior

$$
\begin{aligned}
\operatorname{Pr}\left(\hat{\mathbf{m}},\left\{g_{* L}\right\} \mid\left\{\hat{g}_{L M}\right\}\right) d \hat{\mathbf{m}} & =\operatorname{Pr}\left(\alpha, \beta,\left\{g_{* L}\right\} \mid\left\{\hat{g}_{L M}\right\}\right) d \alpha d \beta \\
& \propto e^{-\chi^{2} / 2} d \hat{\mathbf{m}} .
\end{aligned}
$$


For an isotropic survey (and assumed weak anisotropy), the Fisher matrix is isotropic and we can write $F_{L M, L^{\prime} M^{\prime}}=\delta_{L L^{\prime}} \delta_{M M^{\prime}} / \sigma_{L}^{2}$. Substituting Eq. (17) into Eq. (16) and using the addition theorem for the rotation matrices, we have

$$
\chi^{2}=\sum_{L} \frac{1}{\sigma_{L}^{2}}\left(g_{* L}^{2}-2 g_{* L} \sum_{M} \Re\left[\hat{g}_{L M} D_{M 0}^{L *}(\alpha, \beta, 0)\right]+\sum_{M}\left|\hat{g}_{L M}\right|^{2}\right) .
$$

Noting that $D_{M 0}^{L *}(\alpha, \beta, 0)=\sqrt{4 \pi /(2 L+1)} Y_{L M}(\hat{\mathbf{m}})$, if we define

$$
\hat{g}_{L}(\hat{\mathbf{m}}) \equiv \sum_{M} \hat{g}_{L M} Y_{L M}(\hat{\mathbf{m}})
$$

(i.e. a map of the estimated $\hat{g}_{L M}$ at multipole $L$ ), we can write the posterior as

$$
\begin{aligned}
\operatorname{Pr}\left(\hat{\mathbf{m}},\left\{g_{* L}\right\} \mid\left\{\hat{g}_{L M}\right\}\right) d \hat{\mathbf{m}} \propto \prod_{L} \exp ( & \left.-\frac{2 \pi}{(2 L+1) \sigma_{L}^{2}}\left[g_{* L} Y_{L 0}(\hat{\mathbf{z}})-\hat{g}_{L}(\hat{\mathbf{m}})\right]^{2}\right) \\
& \times \exp \left(\frac{2 \pi}{(2 L+1) \sigma_{L}^{2}} \hat{g}_{L}^{2}(\hat{\mathbf{m}})\right) d \hat{\mathbf{m}} .
\end{aligned}
$$

The marginal distribution for the direction of the axis is given by integrating over $g_{* L}$ :

$$
\operatorname{Pr}\left(\hat{\mathbf{m}} \mid\left\{\hat{g}_{L M}\right\}\right) d \hat{\mathbf{m}} \propto \prod_{L} \exp \left(\frac{2 \pi}{(2 L+1) \sigma_{L}^{2}} \hat{g}_{L}^{2}(\hat{\mathbf{m}})\right) d \hat{\mathbf{m}}
$$

so that contours of constant density are given by the contours of $\sum_{L} \hat{g}_{L}^{2}(\hat{\mathbf{m}}) /\left[(2 L+1) \sigma_{L}^{2}\right]$. For the simple case of a primordial power asymmetry at a single multipole $L$, e.g. a quadrupole asymmetry, $\ln \operatorname{Pr}\left(\hat{\mathbf{m}} \mid\left\{\hat{g}_{L M}\right\}\right)$ is proportional to the square of the map of the reconstructed multipoles.

To illustrate these ideas, in Fig. 5 we plot the marginal distributions for the direction and amplitude of the model analysed in Sec. III A using one of the simulations shown in Fig. 3. We parameterise the direction by the Cartesian components of the equal-area projection $x=$ $2 \sin (\beta / 2) \cos (\alpha)$ and $y=2 \sin (\beta / 2) \sin (\alpha)$. With one year of simulated Planck temperature data, the constraints on the direction of the axis are $\Delta x \approx \Delta y=0.032$ (i.e. $1.8^{\circ}$ ) at $68 \%$ confidence and the $x$ and $y$ components are almost uncorrelated as expected from isotropy. The amplitude $g_{* 2}=0.1 \pm 0.005$ (68\% confidence) with the error being very nearly $\sigma_{2}$. With polarization alone, these constraints weaken to $\Delta x \approx \Delta y=0.10$ (i.e. $5.7^{\circ}$ ) and $g_{* 2}=0.11 \pm 0.02$. The maximum of the posterior in this simulation is at $x=0.042$, $y=-0.035$ and $g_{* 2}=0.099$ ( $T$ only) and $x=-0.033, y=0.062$ and $g_{* 2}=0.11$ ( $E$ only) and the $\chi^{2}$ at these values are 0.223 and 0.236 respectively, with $5-3=2$ degrees of freedom. 

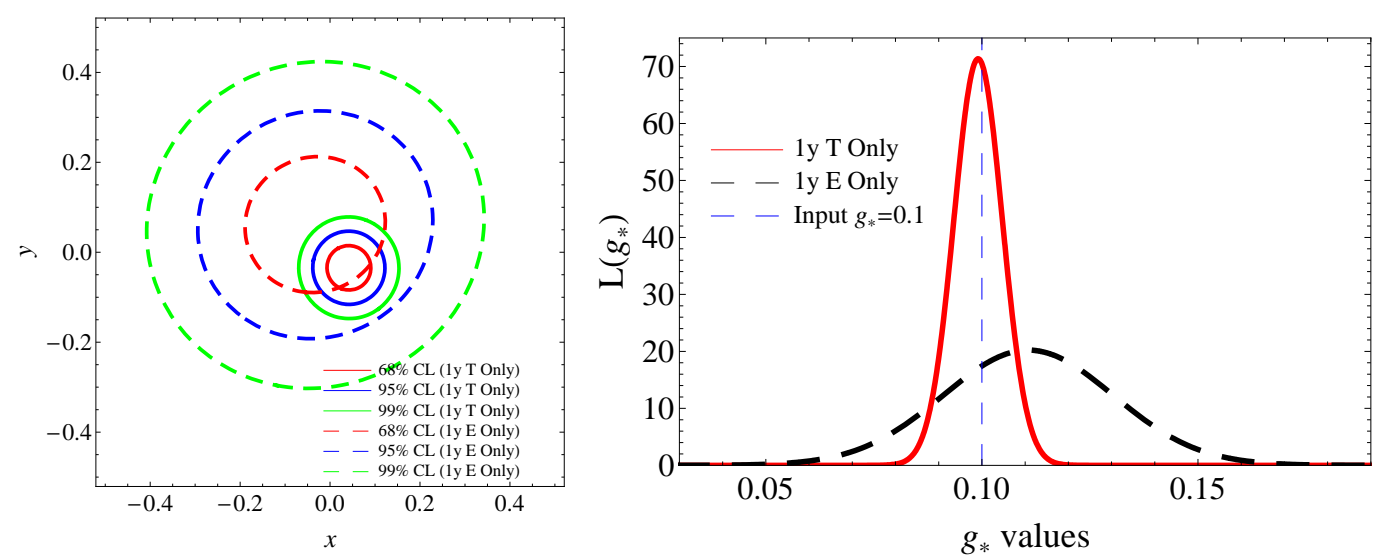

FIG. 5: Marginal distributions for the direction (left) and amplitude $\left(g_{* 2}\right.$, right) from a simulation of the nominal (one-year) Planck survey for a model with an axisymmetric quadrupole asymmetry aligned with the polar axis $\left(g_{L M}=0.1 \delta_{L 2} \delta_{M 0}\right)$. We parameterise the direction with the equalarea projection $x=2 \sin (\beta / 2) \cos (\alpha)$ and $y=2 \sin (\beta / 2) \sin (\alpha)$ and show in solid lines the $68 \%$ [Red], 95\% middle [Blue] and 99\% outer [Green] contours from the temperature alone; dashed-line contours are from the $E$-mode polarization alone. For the amplitude (right), we plot the marginal distributions from $T$ alone (solid line) and $E$ alone (dashed line).

\section{CONCLUSIONS}

As summarized in Sec. I, cosmological perturbations are usually assumed to satisfy statistical isotropy, as expected in simple models of inflation. Yet there is some tentative observational evidence from the CMB suggesting possible departures from statistical isotropy. Here we have shown that Planck should be able to set strong constraints on small departures from statistical anisotropy and, under favourable circumstance, could set constraints on their scale dependence and any preferred direction.

In this paper we have developed and applied quadratic estimators to test for an asymmetry in the primordial power spectrum from temperature and polarization measurements of the CMB. Our estimators are optimal in the limit of isotropic primordial power. We have tested our methods against simulations that include a quadrupole power asymmetry.

We have analysed the ability of the Planck mission to constrain models with quadrupole power asymmetry using temperature and polarization data. Using temperature data alone, Planck should be able to constrain each multipole $g_{2 M}$ of a scale-invariant quadrupole anisotropy at the 0.01 level $(2 \sigma)$, well below the current constraints derived from WMAP 
$\left(\left|g_{2 M}\right|<0.07\right.$ from Ref. [27]). Using polarization data alone from an extended Planck mission (four sky surveys) such an anisotropy can be constrained to an accuracy only about three times worse than from the temperature. This offers the possibility of a consistency check on the existence on any observed departure from statistical isotropy.

If the amplitude of a power asymmetry is large enough, it may be possible to constrain its scale dependence. We have estimated the Fisher errors when additionally constraining a free spectral index describing a power law modulation, $g_{L M}(k)=g_{L M}\left(k_{0} / k\right)^{q}$. For a scale-free quadrupole modulation with an amplitude of $1 \%$ (i.e. $g_{20} / \sqrt{4 \pi}=0.01$ in an axi-symmetric model), we find that an extended Planck mission can constrain the spectral index to a $1 \sigma$ accuracy of $\Delta q \sim 0.3$.

Finally, we have considered the constraints on a preferred direction in models with a purely axisymmetric modulation of the primordial power spectrum. In a scale-free model with a $1 \%$ quadrupole modulation, the direction of the preferred axis can be determined from Planck data to a precision of about $2^{\circ}$ using temperature observations alone and to about $6^{\circ}$ using polarization data alone.

The quadratic estimators developed here for isotropic surveys can, in principle, be straightforwardly extended to deal with real-world effects such as anisotropic noise and Galactic masks. However, the calculation of the $\bar{a}_{l m}^{X}$ in Eq. (11) requires inverse weighting the temperature and polarization data with the full covariance matrix for the anisotropic survey. This has been done for the WMAP temperature data at its native resolution [25, 34] but extending this to polarization and the resolution required for Planck requires further work. In practice, fast estimators can still be constructed for anisotropic surveys, with moderate loss of performance, by replacing $\bar{a}_{l m}^{X}$ with some heuristically-weighted pseudo multipoles (i.e. those computed directly on the masked sky) following techniques used for CMB power spectrum estimation (e.g. see [35] for a review). In either case, care must be taken to subtract the mean-field response - that obtained on average for no primordial power asymmetry - from the quadratic estimator since this is no longer confined to the $L=0$ mode for an anisotropic survey. The mean-field and the estimator normalisation are then generally best determined by Monte-Carlo simulations. 


\section{Acknowledgments}

The authors acknowledge use of the Healpix package and thank Antony Lewis and Duncan Hanson for helpful discussions.

[1] C. L. Bennett et al., ApJS, 148, 1 (2003), astro-ph/0302207.

[2] G. Hinshaw et al., ApJS, 170, 288 (2007), astro-ph/0603451.

[3] M.L. Brown et al., ApJ, 705, 978 (2009), arXiv:0906.1003 [astro-ph].

[4] C.L. Reichardt et al., ApJ, 694, 1200 (2009), arXiv: 0801.1491 [astro-ph].

[5] The ACT Collaboration: J.W. Fowler et al., ApJ 722 (2010) 1148, arXiv:1001.2934 [astro$\mathrm{ph}]$.

[6] N.R. Hall et al., ApJ, 718, 632 (2010), arXiv:0912.4315 [astro-ph].

[7] M. Tegmark, A. de Oliveira-Costa and A. J. S. Hamilton, Phys. Rev. D, 68, 123523 (2003), astro-ph/0302496.

[8] P. Bielewicz, K. M. Gorski and A. J. Banday, Mon. Not. Roy. Astron. Soc. 355, 1283 (2004), astro-ph/0405007.

[9] C. J. Copi, D. Huterer, D. J. Schwarz and G. D. Starkman, Mon. Not. Roy. Astron. Soc. 367, 79 (2006), astro-ph/0508047.

[10] K. Land and J. Magueijo, Phys. Rev. Lett. 95, 071301 (2005), astro-ph/0502237.

[11] F. K. Hansen et al., ApJ 704 (2009) 1448, arXiv: 0812.3795 [astro-ph].

[12] H. K. Eriksen et al., Astrophys. J. 605, 14 (2004), astro-ph/0307507.

[13] M. Cruz et al., ApJ 655, 11 (2007), astro-ph/0603859.

[14] M. Cruz et al., Science 318, 1612 (2007), arXiv: 0710.5737.

[15] C. L. Bennett et al., ApJS 192 (2010) 17, arXiv: 1001.4758 [astro-ph].

[16] C. J. Copi et al., Adv. Astron. Astrophys. 20102010 847541, arXiv: 1004.5602 [astro-ph].

[17] L. Ackerman, S. M. Carroll and M. B. Wise, Phys. Rev. D 75, 083502 (2007), astro-ph/ 0701357.

[18] B. Himmetoglu, C.R. Contaldi, and M. Peloso, Phys. Rev. D 79, 063517 (2009), arXiv: 0812.1231 [astro-ph].

[19] B. Himmetoglu, C.R. Contaldi, and M. Peloso, Phys. Rev. Lett. 102, 111301 (2009), arXiv: 
0809.2779 [astro-ph].

[20] A. L. Erickcek, M. Kamionkowski and S. M. Carroll, Phys. Rev. D. 78, 123520 (2008), arXiv: 0806.0377 [astro-ph].

[21] A. L. Erickcek, C.M. Hirata, and M. Kamionkowski Phys. Rev. D. 80, 083507 (2009), arXiv: 0907.0705 [astro-ph].

[22] C. Gordon, W. Hu, D. Huterer and T. Crawford, Phys. Rev. D 72, 103002 (2005), astro-ph/ 0509301.

[23] C. Dvorkin, H. V. Peiris and W. Hu, Phys. Rev. D 77, 063008 (2008), arXiv: 0711.2321 [astro-ph].

[24] N. E. Groeneboom and H. K. Eriksen, ApJ 690 (2009) 1807, arXiv: 0807.2242 [astro-ph].

[25] D. Hanson and A. Lewis, Phys. Rev. D, 80, 063004 (2009), arXiv: 0908.0963 [astro-ph].

[26] N. E. Groeneboom, L. Ackerman, I. K. Wehus, and H. K. Eriksen, ApJ 722 (2010) 452, arXiv: 0911.0150 [astro-ph].

[27] D. Hanson, A. Lewis, and A. Challinor, Phys. Rev. D., 81, 103003 (2010), arXiv: 1003.0198 [astro-ph].

[28] The Planck Collaboration, 2005, 'The Scientific Programme of Planck', eds. G. Efstathiou, C. Lawrence, and J. Tauber, ESA-SCI(2005), ESA Publications.

[29] K.M. Huffenberger, B.P. Crill, A.E. Lange, K.M. Gorski, C.R. Lawrence, Astron. Astrophys. 510, A58, 2010, arXiv: 1007.3468 [astro-ph].

[30] A. R. Pullen and M. Kamionkowski, Phys. Rev. D 76103529 (2007), arXiv:0709.1144 [astroph].

[31] K. Gorski, E. Hivon, A. Banday, B. Wandelt, F. Hansen, M. Reinecke, M. Bartelman, ApJ, 622, 759 (2005), astro-ph/0409513.

[32] W. Hu and M. White, New Astron. Rev., 2, 323 (1997), astro-ph/9706147.

[33] M. Zaldarriaga, Phys. Rev. D., 55, 1822 (1997), astro-ph/9608050.

[34] K. M. Smith, O. Zahn, O. Doré, Phys. Rev. D., 76, 043510 (2007), arXiv:0705. 3980 [astro$\mathrm{ph}]$.

[35] G. Efstathiou, Mon. Not. Roy. Astron. Soc., 349, 603 (2004), astro-ph/0307515. 